density (QID) was CPS minus TPS. TPS, CPS, and QID ability to predict ORR (ROC analysis) and overall survival (OS) hazard ratio (HR) (pembrolizumab vs chemotherapy) were evaluated.

Results: With pembrolizumab, area under ROC curve $(95 \% \mathrm{CI})$ was $0.69(0.58-0.80)$ for CPS, $0.67(0.56-0.77)$ for QID, and $0.55(0.46-0.64)$ for TPS. At each cutoff, QID had lower sensitivity and Youden Index and larger OS HR than CPS. QID appeared to be orthogonal to TPS.

Conclusions: Trends suggest tumour cell expression is an important component of PD-L1 as a predictive biomarker of pembrolizumab in mTNBC. When immune cells alone were used, a meaningful number of responders was missed, and OS benefit trended toward higher HR estimates.

\section{PAN-TUMOUR ANALYSIS OF THE ASSOCIATION BETWEEN PD-L1 COMBINED POSITIVE SCORE (CPS) AND RESPONSE TO PEMBROLIZUMAB MONOTHERAPY}

Lingkang Huang, Jared Lunceford, Junshui Ma,

Kenneth Emancipator

Merck \& Co Inc, Kenilworth, NJ, USA

PD-L1 is expressed on tumour and immune cells; PD-L1 CPS captures tumour and immune cell expression in one aggregate score. We performed a retrospective, exploratory analysis of CPS as an enrichment biomarker of pembrolizumab monotherapy across multiple tumour types. PD-L1 expression was assessed using PD-L1 IHC 22C3 pharmDx and measured using CPS (number of PD-L1-staining cells [tumour cells, lymphocytes, macrophages] divided by total number of tumour cells, multiplied by 100). Data were pooled from 11 studies across tumour types for pembrolizumab and for standard-of-care (in controlled studies). Estimates of ORR, prevalence, and receiver operating characteristics (ROC) analysis was performed over various CPS cut-points. CPS distribution by response, tumour type, and line of therapy were also assessed. 3769 patients had available PD-L1 CPS (pembrolizumab, $n=2678$; standard-of-care, $n=1091$ ). Area under the ROC curve for ORR was 0.63 (95\% CI, 0.61-0.66; pembrolizumab) versus 0.48 (95\% CI, 0.43-0.53; standard-ofcare); cut-points of $1,10,20$, and 50 were also examined. This pan-tumour analysis demonstrates that CPS is an effective method for scoring PD-L1 expression and can be used as a predictive biomarker to identify patients likely to respond to pembrolizumab monotherapy. CPS demonstrated enrichment of response to pembrolizumab monotherapy across most tumour types.

\section{INTERNAL AUDIT OF TWO ANTINEURONAL IMMUNOBLOTS AND THE ROLE OF INDIRECT IMMUNOFLUORESCENCE IN SAMPLE SCREENING}

L. S. Gardner $^{1,2}$, S. Culican ${ }^{1}$, D. Campbell ${ }^{1}$, M. Dela Cruz ${ }^{1}$,

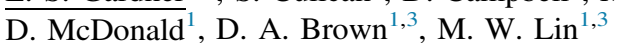

${ }^{1}$ Immunopathology Department, ICPMR, Westmead Hospital, Sydney, NSW, Australia; ${ }^{2}$ Faculty of Medicine, University of Queensland, Qld, Australia; and ${ }^{3}$ Faculty of Medicine, University of Sydney, NSW, Australia

Anti-neuronal antibodies are associated with a range of clinical phenotypes that are often preceded by malignancy. Most of these antibodies can be identified by indirect immunofluorescence
(IIF) using commercial primate neural tissue slides. The EUROImmun 12 and upgraded RAVO 14 antigen line immunoblots were directly compared to replace a 9 antigen RAVO immunoblot. Initial assessment was with samples with known positive specificities by IIF. The second phase involved parallel testing of routine pooled negative IIF samples as per institutional standard antineuronal testing algorithm. IIF results and clinical correlation were used to arbitrate on discordant immunoblot results. Correlation in known positives was $80 \%$. The EUROImmun immunoblot did not identify disease causing SOX1 and Recoverin antibodies. Routine pooled analysis did not identify discrepancy between the platforms. Assessment of 318 patient samples did not reveal any cases where a diagnosis was correctly identified by line immunoblot and not IIF. A single sample with positive Yo antibodies was identified by both platforms without clinical or IIF correlation, making this a likely false positive. Subsequent to this audit, Westmead ICPMR has migrated to the 14 antigen RAVO line blot for confirmation of positive IIF or for patients with equivocal results only.

\section{CHALLENGES OF PRODUCING A NEW EXTERNAL QUALITY ASSURANCE PROGRAM - A PROCESS IMPROVEMENT PROJECT}

\section{Grace Moyo, Loriza Khan}

Royal College of Pathologists of Australasia Quality Assurance Programs (RCPAQAP), St Leonards, Sydney, Australia

A requirement for validation and accreditation of a new test in many laboratories is the availability of an accredited external quality assurance (EQA) program. RCPAQAP conducted a process improvement project to reduce the turnaround time to introduce a new accredited EQA program. A voice of customer (VoC) survey was distributed to identify customer requirements for EQA availability at the time of test verification. Data was collected for 13 serology and immunology EQA programs dating from 2006. Total cycle time for introducing a new program was captured in days. $73 \%$ of customers required a new EQA program to be available in 6 months or less of a test becoming available. The median number of days to achieve accreditation was 1427 days. Three areas of improvement were identified. These were (1) time taken to select a program to pilot, (2) time taken to source clinically appropriate samples, and (3) the time taken to gather program-specific information regarding the new test. The process improvement project has allowed RCPAQAP to understand customer needs and the complexities of planning a new EQA program better and develop processes to deliver superior EQA programs to advance outcomes in patient care and promote excellence in laboratory output.

\section{ARTHROGRAPHIS KALRAE PROSTHETIC JOINT INFECTION: CASE REPORT AND REVIEW OF THE LITERATURE}

Lucy C. Crawford $^{1,2}$, Sarah E. Kidd ${ }^{2,3}$, Tom Savvoulidis ${ }^{4}$, Renjy Nelson ${ }^{2,}$

${ }^{1}$ Microbiology and Infectious Diseases Directorate, SA Pathology, Adelaide, SA, Australia; ${ }^{2}$ Faculty of Health Sciences, University of Adelaide, SA, Australia; ${ }^{3}$ National Mycology Reference Centre, SA Pathology, Adelaide, SA, Australia; ${ }^{4}$ Medacta International, Australia; and ${ }^{5}$ Infectious 